\section{Regards sur l'économie allemande}

Bulletin économique du CIRAC

$87 \mid 2008$

Varia

\title{
"Considérer la Chine avec le respect dû à un partenaire »
}

\section{Monika Stärk et Isabelle Bourgeois}

Traducteur : Isabelle Bourgeois

\section{OpenEdition \\ Journals}

Édition électronique

URL : http://journals.openedition.org/rea/970

DOI : $10.4000 /$ rea. 970

ISBN : 978-2-8218-0870-6

ISSN : 1965-0787

Éditeur

CIRAC

Édition imprimée

Date de publication : 1 juillet 2008

Pagination : 13-26

ISSN : 1156-8992

\section{Référence électronique}

Monika Stärk et Isabelle Bourgeois, « «Considérer la Chine avec le respect dû à un partenaire » »,

Regards sur l'économie allemande [En ligne], 87 | juillet 2008, document 2, mis en ligne le 01 juillet 2010, consulté le 01 mai 2019. URL : http://journals.openedition.org/rea/970 ; DOI : 10.4000/rea.970 


\title{
"Considérer la Chine avec le respect dû à un partenaire "
}

\author{
Un entretien avec Monika Stärk
}

La Chine est aujourd'hui le premier partenaire commercial de l'Allemagne et de I'UE en Asie (voir dans ce numéro). Si les échanges germano-chinois ont été dynamisés depuis les années 1980 par l'ouverture de l'économie chinoise et l'entrée de la République populaire dans I'OMC, ils retrouvent aujourd'hui, après une histoire mouvementée, des niveaux comparables à ceux du début du XX $X^{e}$ siècle, c'est-à-dire durant la première phase de la mondialisation après l'émergence de l'ère industrielle. Ainsi, en 1913, la Chine réalisait $9 \%$ de ses échanges avec l'Allemagne. $25 \%$ des biens exportés dans le monde par la Chine et $19 \%$ des biens qu'elle importait transitaient par les maisons de commerce allemandes, principalement sises à Hambourg où est restée vivace la culture de la Hanse, cette zone de libre-échange née à la fin du XIIF siècle dans l'espace baltique et dont la guerre de Trente ans scella la fin au XVIF. Alors que la majeure partie des échanges s'effectuait par la suite dans le bassin de la Méditerrannée, la découverte des Amériques les réorienta vers les relations transatlantiques. L'industrialisation et l'internationalisation des échanges qu'elle permit scellèrent les liens intercontinentaux qui perdurent jusqu'à aujourd'hui, tout en permettant l'ouverture sur le Pacifique. De retour en 1929 d'un voyage en Asie, le chimiste Carl Duisberg, alors P.D.G. d'IG Farben (le groupe BASF renaîtra de ses cendres après 1945), constatait dans les colonnes de l'Ostasiatische Rundschau, la revue de l'association Ostasiatischer Verein, que « lentement mais sûrement, le centre de gravité économique du monde se déplace de l'Atlantique vers le Pacifique " (cité in Eberstein, 2000). L'ouverture du commerce mondial, la globalisation des activités et la montée en puissance plus récente de la Chine valident son analyse.

Les ruptures des relations diplomatiques et les conflits mondiaux du XX siècle ont souvent interrompu les échanges commerciaux avec la Chine, mais jamais durablement. Les liens se sont vite renoués, précédant la reprise des contacts officiels. Un rôle clef dans le développement des relations commerciales germanochinoises revient à l'Ostasiatischer Verein (OAV), un lobby créé en 1900 par des commerçants hambourgeois pour défendre le libre-échange avec l'Asie orientale, dont la Chine. L'OAV a aujourd'hui une fonction éminente de conseil pour les entreprises allemandes désireuses de s'implanter en Chine. Nous avons demandé à Monika Stärk, membre du bureau exécutif de l'OAV depuis 2003 et membre ès qualités du comité Asien-Pazifik-Ausschuss, plateforme des activités asiatiques des grandes fédérations professionnelles et des chambres de commerce allemandes, de nous présenter l'approche allemande des relations commerciales avec la Chine depuis son entrée dans l'OMC.

\section{Une devise : «le changement par le commerce »}

Depuis la visite officielle du Dalaï Lama en Allemagne à l'automne 2007, les relations diplomatiques entre la République populaire de Chine et la République fédérale d'Allemagne se sont nettement refroidies. Les relations commerciales s'en trouventelles affectées?

Le tremblement de terre de ce printemps dans la Province du Sichuan a relégué le Tibet au second plan des préoccupations de l'opinion allemande; mais la question des droits de l'Homme resurgira à l'approche des Jeux Olympiques.

L'opinion publique méconnaît l'interdépendance économique entre l'UE et la Chine 
Le dialogue et l'exemple sont plus fructueux que les leçons de morale
Cela dit, l'impact du refroidissement des relations diplomatiques sur les échanges commerciaux s'est jusqu'ici tout au plus manifesté de manière indirecte, et dans quelques rares cas isolés seulement. A moyen terme toutefois, si la controverse médiatique prenait le pas sur le dialogue constructif, la position des acteurs économiques allemands dans la République populaire pourrait s'en trouver affectée. L'opinion publique allemande ne mesure pas encore l'étroitesse des liens d'interdépendance entre l'Europe et la Chine, ni l'importance qui revient à un partenariat pour répondre aux défis d'une économie mondiale globalisée. Trop peu informée encore sur ces réalités, elle préfère cultiver ses peurs face à la mondialisation et accuser la Chine de tous les maux.

Jürgen Hambrecht, P.D.G. de BASF et président de la Commission Asie-Pacifique (Asien-Pazifik-Ausschusses der Deutschen Wirtschaft, APA) qui fédère les intérêts de l'économie allemande dans la région, plaide pour une approche des relations avec la Chine qu'il résume ainsi : « le changement par le commerce » (Wandel durch Handel). L'Ostasiatischer Verein partage-t-il cette approche?

Non seulement mon association soutient pleinement cette approche, mais elle a pris une part active dans la formulation de cette politique. Les milieux économiques allemands ne considèrent pas les droits de l'Homme comme une question secondaire, bien au contraire : ces derniers sont un facteur essentiel de la culture comme de la gouvernance d'entreprise chez nous. Lorsqu'elles implantent des sites de production en Chine, les sociétés allemandes y exportent tout naturellement l'éthique et les standards en vigueur en Allemagne, que ce soit en matière de conditions de travail, de protection sociale ou de préoccupations environnementales. Ce faisant, elles apportent une contribution non négligeable au processus de transformation de la société chinoise.

Pour en revenir à la question des libertés : depuis de nombreuses années, l'amélioration de la situation est régulièrement revendiquée dans le cadre d'un dialogue bilatéral entre l'Allemagne et la Chine, et cette insistance porte ses fruits. II faut évoquer ici par exemple le Dialogue germano-chinois sur l'Etat de droit (Deutschchinesischer Rechtsstaatsdialog), institué en 1999 sous le chancelier Schröder et qui réunit régulièrement les deux gouvernements, ou encore le Forum de dialogue germano-chinois (Deutsch-chinesisches Dialogforum) institué en 2005 par les présidents $\mathrm{Hu}$ Jintao et Horst Köhler et qui réunit à intervalles réguliers des experts de haut niveau sous la direction de Xu Kuangdi, vice-président de la Chinese People's Consultative Conference et de Heinrich von Pierer, anciennement à la tête de Siemens. Ces deux plateformes d'échanges sont un haut lieu de dialogue constructif où peuvent être soulevées des questions délicates - en toute sérénité, car loin des caméras.

Par définition, la communication par l'échange, par le dialogue, s'avère bien plus fructueuse que les controverses médiatisées et leurs arguments sans nuances. Car il ne faut pas oublier non plus que, en matière de libertés, la Chine a réalisé des progrès considérables au cours de ces dernières années, indissociables d'une mutation extraordinairement profonde de la société, et dont l'effet a été de sortir de la misère des millions de Chinois.

L'ex-chancelier Helmut Schmidt (SPD) ne cesse de rappeler aux milieux politiques qu'il ne revient pas à l'Allemagne de « s'immiscer» dans les affaires intérieures de la Chine. Dans un discours tenu le 12 juin à la Deutsche Gesellschaft für Auswärtige Politik, la veille du voyage officiel du ministre fédéral des Affaires étrangères dans la République populaire, il a évoqué le bref passé colonial de l'Empire allemand pour délivrer ce message : "Nous devons nous garder de retomber dans cette attitude moralisatrice qui caractérisait l'ère de Guillaume II ". C'est donc cela que signifie aussi votre devise du « changement par le commerce »?

Le commerce des idées nous amène aussi à changer notre vision du monde
Nous sommes convaincus que c'est là la meilleure approche, même si elle ne porte ses fruits qu'à long terme. La communication, le dialogue, les échanges commerciaux et le commerce des idées se sont avérés plus judicieux que les sanctions ou le boycott. Cela vaut a fortiori pour un pays qui, comme la Chine, progresse continûment dans le sens des libertés et où rien n'est plus inapproprié, 
mal venu voire contreproductif que la critique permanente ou une attitude moralisatrice. Plus généralement, alors que le contexte mondial dans lequel nous évoluons subit de profondes mutations non seulement économiques, mais aussi géopolitiques, il faut que nous fassions évoluer nous aussi notre démarche, que nous soumettions à une analyse critique la manière dont nous abordons des cultures, des systèmes étrangers. Car ce n'est qu'ainsi que nous parviendrons à trouver la manière la plus adéquate de promouvoir nos propres valeurs et ainsi de faire valoir nos intérêts.

Certes, le dialogue est stratégique pour une économie aussi ouverte que celle de l'Allemagne. Mais certains n'hésitent pas à y déceler comme une dose d'hypocrisie : si l'Allemagne défendait des positions plus fermes, ne risquerait-elle pas de mettre en danger cet 'établi prolongé' qu'est le site de production chinois ?

La Chine ne mérite plus cette appellation. Elle n'est plus seulement ce site externalisé à bas salaires où les entreprises allemandes faisaient produire des biens destinés à l'exportation vers les marchés de l'occident industrialisé. Cette époque-là est pratiquement révolue. Mais il faut rappeler aussi à ces observateurs critiques qu'ils ont la mémoire courte : car durant de longues années, la production chinoise à bas coûts a permis de contenir l'inflation au sein de l'Union européenne et, dans le même temps, de garantir le niveau de vie élevé des Européens en leur permettant d'acheter au meilleur prix des produits textiles et même des biens de consommation de qualité technologique supérieure.

Les entreprises allemandes considèrent aujourd'hui la Chine comme un marché d'importance stratégique. Car c'est, entre autres facteurs, de la réussite du positionnement sur ce marché que dépendra l'évolution de l'économie européenne. Cela est particulièrement vrai eu égard à la rapidité fulgurante des progrès technologiques réalisés par la Chine, qu'ils soient le résultat d'un effort d'innovation propre ou d'un transfert de technologies imposé. Par ailleurs, dans un nombre croissant de domaines, la Chine se positionne en concurrent des économies européennes sur l'échiquier mondial. La présence d'entreprises allemandes sur ce marché est dès lors impérative pour préserver la compétitivité de l'Allemagne et de l'Europe, ainsi que pour participer au développement de standards conjoints. Cultiver et développer le partenariat avec la Chine est aujourd'hui une priorité dans l'intérêt de l'Empire du Milieu comme de ses partenaires mondiaux.

Est-ce cela qu'entendait le président de la République fédérale, Horst Köhler, lorsqu'il évoquait, dans un discours tenu il y a deux ans devant votre association, le fait que « l'Asie change la face du monde»?

La montée en puissance de l'Asie change effectivement la donne mondiale. Or une grande partie de la société allemande est mal préparée pour comprendre ces évolutions et appréhender les nouveaux défis qu'elles impliquent. En réaction à ces changements, certaines voix s'élèvent pour réclamer toutes sortes de barrières protectionnistes. Rien n'inquiète plus les milieux économiques allemands, et les sociétés traitant avec l'Asie se sont prononcées fermement contre toute mesure protectionniste. Eriger des barrières pour limiter les importations ou les investissements permet uniquement de calmer momentanément l'angoisse des Européens face à la globalisation. A moyen terme, de telles mesures mettraient en danger la compétitivité de l'économie allemande et européenne, et donc les fondements mêmes de notre prospérité. C'est à une autre méthode qu'il faut recourir pour endiguer cette angoisse : il faut exposer plus clairement dans l'espace public les perspectives qu'ouvre la coopération économique avec l'Asie, et le bénéfice qu'en tire l'économie mondiale. Cet effort didactique est stratégique pour l'Allemagne, dont l'économie ouverte s'est déjà bien adaptée à la globalisation et a tout à y gagner.

Le président Köhler avait tenu son discours lors d'un « Liebesmahl 》 organisé par votre association en mars 2006. De quoi s'agit-il ?

C'est la grande manifestation annuelle organisée par l'OAV et les entreprises allemandes présentes en Asie orientale autour de l'intervention de personnalités
La Chine est aujourd'hui un concurrent à part entière de l'UE

Nos opinions publiques sont mal préparées encore à la montée en puissance de l'Asie 
L'appui politique est précieux, mais pas indispensable

L'Allemagne mène une campagne d'image en Chine des milieux économiques et politiques, issues d'Europe et d'Asie, au cours d'un grand dîner. Son nom est emprunté à une vieille tradition chrétienne : celle de l'agape - littéralement : le repas d'amour -, où se réunissait la communauté des croyants. La marine prussienne avait renoué avec cette tradition au XIX ${ }^{e}$ siècle lorsqu'elle accostait en Asie ; les officiers invitaient alors à bord les commerçants allemands installés dans la région pour un repas confraternel, appelé «agape est-asiatique »(Ostasiatisches Liebesmahl). Or l'Ostasiatischer Verein avait été créée en 1900 par des commerçants allemands à leur retour d'Asie; ils ont en quelque sorte ré-importé cette coutume qui fait partie depuis de la tradition de notre association, même si cette Agape a évolué avec le temps.

\section{La présence allemande en Chine est auto-organisée et suit une logique de réseaux de partenariats}

Les relations économiques entre l'Allemagne et la Chine doivent beaucoup au chancelier Schröder qui a effectué plusieurs voyages officiels dans la République populaire. Michael Glos, l'actuel ministre fédéral de l'Economie, s'y est rendu lui aussi plusieurs fois, accompagné d'une délégation d'hommes d'affaires. Les entreprises allemandes sont-elles tributaires de l'art de la négociation diplomatique pour réussir sur le marché chinois?

Un encadrement politique ou diplomatique est précieux lorsqu'il s'agit de la passation de marchés publics. II est bénéfique également aux entreprises qui n'œuvrent pas directement dans les secteurs publics ou parapublics: le fait qu'elles fassent partie d'une délégation politique de haut rang leur confère en effet un grand prestige aux yeux de leurs partenaires comme de leurs collaborateurs chinois. Certes, en Chine et dans la plupart des autres pays asiatiques, le protocole joue un rôle plus déterminant qu'en Europe. Et pour une entreprise allemande, le fait d'être membre d'une délégation officielle de haut rang apporte un sucroît de prestige. Mais en Allemagne aussi, le fait qu'un responsable politique honore de sa présence une manifestation organisée par une entreprise est un gain inestimable en termes d'image. Or si une approche protocolaire est incontournable pour les projets de coopération publics, il convient de ne pas généraliser ; la plupart du temps, les entreprises agissent seules, et avec succès. Cela vaut particulièrement pour les représentants des grands groupes cotés au DAX qui voient s'ouvrir les portes des administrations et des ministères sans appui politique. L'accès étant moins facile pour les PME, elles gagnent en réputation si elles font partie d'une délégation officielle.

La République fédérale a lancé en 2007 une campagne qui culminera lors de l'Exposition universelle de Shanghai en 2010 : "L'Allemagne et la Chine avancent de conserve " (Deutschland und China - Gemeinsam in Bewegung)...

... cette campagne n'a pas pour priorité la promotion du commerce extérieur allemand. II s'agit plutôt d'une longue série de manifestations organisées conjointement par les milieux politiques, économiques et culturels, avec pour objectif de faire connaître l'Allemagne en tant que site d'innovation. Elle vise aussi à la présenter dans l'opinion chinoise comme un partenaire avec qui résoudre les grands défis que sont l'urbanisation et le développement durable. La campagne vise un public large, à la fois par la concentration sur les villes moyennes et par l'orientation de son contenu. Elle ne se contente en effet pas d'offrir aux élites des manifestations prestigieuses organisées par des institutions culturelles de renom comme les grands orchestres et musées ; elle intègre la culture pop et offre des plateformes de dialogue et de rencontre pour tous, ainsi par exemple un atelier d'expérimentation chimique s'adressant aux enfants. La campagne se révèle un franc succès. Ce qui la distingue en revanche de celles menées par d'autres Etats membres de l'UE, c'est la part relativement faible du financement public dont elle bénéficie, les entreprises y apportant une contribution majeure. II faut dire aussi à la décharge des pouvoirs publics que l'Allemagne s'est dotée d'un dispositif conséquent pour promouvoir son commerce extérieur. 

Comment s'articule le dispositif public de soutien au commerce extérieur avec l'ini-
tiative des entreprises?

Le soutien allemand au commerce extérieur présente une structure très diversifiée. L'élément clef du dispositif est sans nul doute la facilité d'accès à l'information dont bénéficient les entreprises grâce aux structures étatiques comme l'Agence fédérale pour le commerce extérieur (Bundesanstalt für Außenwirtschaft, Bfai) ou aux chambres de commerce extérieures, les Auslandshandelskammern (AHK). Ces institutions ont des liens étroits avec les fédérations professionnelles des acteurs économiques. Parmi la vaste gamme des aides qui existent en Allemagne, les plus importantes sont en l'occurrence les garanties d'investissement et les garanties publiques à l'export. En ce qui concerne les premières (elles couvrent les risques politiques), deux destinations sont prioritaires: la Chine et la Russie. En 2007, par exemple, pour la seule Chine, le montant des garanties s'est élevé à 713 millions $€$ pour 36 projets d'investissement. Or pour l'instant, seuls les grands groupes allemands ont recours systématiquement à ces garanties; dans le Mittelstand, seule une PME sur trois en bénéficie, et il faut impérativement y accroître la diffusion de ce type de soutien.

En matière de garanties publiques à l'export aussi, la Chine occupe une place centrale : le montant des garanties Hermes allouées en 2006 pour cette destination a dépassé 1,3 milliard $€$. Mais ici aussi, il est stratégique d'en étendre l'accès aux PME qui manquent d'informations sur ces crédits ou que leur coût administratif effraie. C'est la raison pour laquelle vient d'être développée une version 'light', à charge bureaucratique nettement réduite, des crédits Hermes destinés aux opérations moins volumineuses d'exportation. Outre ces aides publiques, il existe une offre privée florissante de garanties à l'exportation. D'une manière générale, l'ensemble de ce riche dispositif de soutien au commerce extérieur reste trop fermé encore aux PME qui tout simplement n'ont pas les moyens humains ni le temps pour y recourir systématiquement. La priorité est aujourd'hui de faciliter l'accès à ce dispositif aux PME qui n'y ont encore jamais eu recours, car ce n'est qu'une fois qu'une entreprise a pu bénéficier de ces mesures qu'elle en comprend réellement l'utilité : elles atténuent les risques, facilitent la marche opérative des affaires et permettent ainsi, in fine, d'économiser les ressources.

Ce dispositif est si complexe, les interlocuteurs si nombreux, que les Allemands envient parfois leurs homologues français et leurs structures centralisées...

... le fait que les ambassades de France soient l'interlocuteur central du dispositif d'expansion économique présente en effet l'avantage indéniable d'une grande lisibilité, en comparaison de l'éclatement allemand des compétences entre les ambassades, les chambres de commerce AHK et l'Agence fédérale Bfai. Mais une restructuration, notamment sous la forme d'un pilotage plus étroit par la Bfai et les AHK, est actuellemnt à l'étude. Cela dit, la pluralité du dispositif allemand présente aussi des avantages : comme il ne se concentre pas exclusivement sur les besoins des grands groupes publics ou para-publics, il permet d'offrir aux nombreuses PME présentes à l'international ces prestations sur mesure qui leur sont nécessaires. A cela s'ajoute un autre élément structurel qui fait la force du dispositif allemand: l'implication directe des entreprises dans l'organisation comme l'action des chambres de commerce extérieures. Certes, notre dispositif de soutien au commerce extérieur s'est structuré au fil de l'Histoire, ce qui exige désormais sa modernisation, et notamment une réorganisation partielle des diverses compétences. Mais au cours des dernières années, diverses initiatives ont été menées avec succès pour en accroître l'efficience et le rapprocher encore plus des préoccupations des entreprises. II n'en reste pas moins que, bien qu'il soit perfectible, le dispositif s'avère toujours foncièrement efficient.

On présente parfois l'Asien-Pazifik-Ausschuss (APA) créé en 1993 comme le 'poste d'expansion économique' de la Fédération allemande de l'Industrie (BDI)...

... ce n'est pourtant pas le cas. La confusion vient de ce que le BDI assure une fonction de coordination des intérêts industriels au sein de l'APA. II n'en est pas
Interpénétration étroite entre structures publiques et représentations professionnelles

Les PME restent le parent pauvre du soutien au commerce extérieur

Un dispositif de soutien efficient malgré des structures éclatées

Les acteurs économiques présents en Asie ont leur lobby : l'APA... 
.... qui défend l'intérêt général du site Allemagne

pour autant le représentant de ces intérêts à l'international. En réalité, l'AsienPazifik-Ausschuss réunit dans une plateforme commune d'une part les grandes fédérations professionnelles et chambres consulaires allemandes : la fédération des chambres de commerce et d'industrie (DIHK), de même que celles de l'industrie (BDI), des banques (Bankenverband) ou du commerce extérieur (BGA). D'autre part, elle compte parmi ses membres fondateurs l'association de spécialistes de l'Asie qu'est l'Ostasiatischer Verein. La mission de l'APA est d'offrir aux acteurs économiques une plateforme commune chargée de représenter leurs intérêts auprès des milieux politiques et de les défendre dans tous les dossiers d'intérêt général. Autrement dit: les intérêts de l'économie allemande en Asie s'expriment par un seul canal.

Pour éviter justement les risques de confusion avec l'action menée dans la réalité quotidienne par les diverses organisations professionnelles, nous avons adopté au sein de l'APA une description officielle de son positionnement à l'échelon où se définissent les grandes orientations politiques qui déterminent le cadre des activités. A savoir celle-ci : depuis 1993, l'Asien-Pazifik Ausschuss est le porteparole des acteurs économiques allemands présents en Asie pour les pouvoirs publics allemands comme pour leurs homologues dans les pays partenaires dans cette région ; l'APA est également une instance où sont formulés et débattus les intérêts respectifs dans le domaine de la politique économique. Quant aux prestations concrètes liées au commerce extérieur, elles continuent de relever des compétences exclusives des fédérations qui sont ses membres fondateurs, de même que des associations régionales ou sectorielles qu'elles fédèrent.

\section{Ostasiatischer Verein. Le commerce résiste aux aléas de l'Histoire.}

L'association OAV a été créée le 13 mars 1900 pour défendre les intérêts des «pionniers d'Asie ", ces commerçants allemands qui développaient les échanges dans la région depuis l'intensification des échanges mondiaux dans la dernière décennie du $\mathrm{XIX}^{\mathrm{e}}$ siècle. Dans cette première phase de la mondialisation liée à l'essor de l'ère industrielle, et qui a incité l'Allemagne de Guillaume II à concurrencer les empires coloniaux britannique et néerlandais, l'Asie était une région stratégique pour l'accès à la matière première la plus recherchée alors : le caoutchouc. C'est plus tard seulement que les entreprises allemandes penseront à installer des sites de production au Japon et en Chine, avant de rivaliser avec leurs concurrents et les Etats-Unis pour y ouvrir des succursales bancaires.

Depuis sa création, l'OAV défend le principe du libre-échange, en franche opposition aux protectionnismes d'inspiration bismarckienne, puis hitlérienne. A plusieurs reprises dans l'histoire mouvementée des relations politiques intercontinentales, l'association a permis de préserver les échanges économiques entre l'Allemagne et l'Asie, base sur laquelle ont pu être renouées ensuite les relations diplomatiques. L'OAV a ainsi toujours eu un rôle d'intermédiaire.

Cette sorte de primauté de l'économique sur le politique s'était affirmée à l'issue de la Première Guerre mondiale : « la politique asiatique de l'Allemagne ne pouvait [en effet] être qu'à dominante économique » (Eberstein, 2000) puisque le Traité de Versailles avait définitivement mis fin à l'empire colonial allemand. A l'issue de la Deuxième Guerre mondiale, les relations avec l'Asie sont au point mort, et l'économie allemande n'est plus représentée dans la région. La base des activités se reconstitue sur le sol allemand, à Hambourg, lorsque les Alliés autorisent l'ouverture d'une liaison maritime vers l'Asie orientale (1953 ; seuls sont desservis les ports de Hongkong et de Taiwan). En 1954, grâce au travail de lobying de l'OAV notamment, la République populaire de Chine ouvre ses ports à la marine marchande allemande, et les relations commerciales reprennent en 1955. Au printemps 1957, l'OAV organise dans le cadre de la foire de Hanovre une journée consacrée à l'Asie du sud-est et, le 27-09-1957, l'Ost-Ausschuss der deutschen Wirtschaft (précurseur de l'APA) signe avec le Comité de promotion du commerce international chinois (CCPIT) un accord sur la reprise des relations commerciales. Ce n'est que 16 ans plus tard, en 1973, que la République fédérale et la République populaire signeront un acord commercial officiel.

Si aujourd'hui, l'OAV défend les intérêts de l'ensemble de l'économie allemande présente en Asie, elle a été d'abord le lobby du seul commerce. Du fait du boom de l'industrie allemande au tournant du XIX et du XX ${ }^{e}$ siècle, les industriels n'ont eu de cesse de se faire représenter par les commerçants, mais la divergence des intérêts entre ces deux types d'activité a longtemps été source de tensions internes, les industriels cherchant à créer leur propre représentation. L'affaire s'est réglée à la fin des années 1950, par une répartition du travail 'à l'allemande' : en 1952, le gouvernement fédéral incite les fédérations de l'industrie (BDI) et des chambres de commerce (DIHT) à s'associer avec l'OAV pour créer l'Ost-Ausschuss der deutschen Wirtschaft, un comité officiel chargé des relations commerciales avec les pays de l'Est et la Chine. La Chine, qui avait réorienté le flux de ses échanges vers l'URSS et avait été placée sous embargo par l'ONU, ne figurait donc pas dans cette zone. L'Allemagne ne pouvant nouer des relations officielles avec la Chine, ce comité instaure en son sein un groupe de travail sur la Chine, dirigée en union personnelle par l'OAV.

L'OAV étant de statut privé et ne représentant aucun intérêt politique officiel, il a pu relancer les échanges avec la République populaire. Depuis, les compétences régionales du comité, rené sous le nom de Asien-Pazifik-Ausschuss der deutschen Wirtschaft (APA) en 1993, se sont étendues à l'ensemble de la région asiatique. Quant à l'OAV, il continue d'assurer une mission d'interface entre milieux économiques et politiques, en participant par exemple à l'organisation de voyages officiels. De par sa mission, il œuvre principalement sur le sol allemand, son siège étant toujours à Hambourg ; il dispose certes d'une représentation en Asie, mais celle-ci est sise au Myanmar (Birmanie). (IB) 
L'OAV est une structure de droit privé, créée à l'initiative de commerçants de Hambourg et de Brême lors de l'émergence de l'ère industrielle et de la mondialisation. Quelle est la mission de votre association aujourd'hui ?

L'Ostasiatischer Verein a été fondé en 1900 par des 'pionniers de l'Asie' : les grandes maisons de commerce des villes hanséatiques de Brême et de Hambourg. II est donc né de l'initiative privée, et sa mission est restée inchangée depuis : il s'agit d'un réseau chargé de défendre les intérêts de l'économie auprès du monde politique en Allemagne et en Asie. Au fil du temps, la structure des membres de l'OAV s'est diversifiée, et notre association fédère désormais des entreprises issues de toutes les branches et situées dans toute l'Allemagne. Nous apportons donc notre soutien à toutes les sociétés désireuses d'engager des relations avec l'Asie. Nous conseillons aujourd'hui surtout les PME industrielles des grands sites de production que sont le Bade-Wurtemberg, la Bavière, le Rhénanie-Palatinat ou la Rhénanie du Nord-Westphalie, et qui, lorsqu'elles prennent l'initiative de s'engager en Asie, recourent à notre réseau d'expertise comme à notre offre d'accompagnement et de conseil. II faut insister sur ce point : l'OAV est depuis toujours resté une initiative privée, indépendante de toute influence politique, et se conçoit comme un réseau d'expertise pour l'ensemble du monde asiatique. C'est-à-dire que nous ne nous concentrons pas seulement sur les 'poids lourds' de la région, comme la Chine, mais que nous intégrons aussi dans nos activités les pays moins développés au contexte politique plus délicat. C'est là que réside notre continuité séculaire : nous sommes restés des pionniers, notre rôle consiste toujours à ouvrir des portes aux entreprises allemandes. Simplement, aujourd'hui, nous nous plaçons au service de l'économie allemande dans son entier.

Votre association n'en est pas moins restée solidement ancrée dans la métropole portuaire de Hambourg...

Les villes hanséatiques de Hambourg et de Brême étaient la plateforme du commerce extérieur allemand et donc le site des entreprises qui ont développé les échanges avec l'Asie au début du $X X^{e}$ siècle. A l'origine, ces échanges étaient l'affaire des seuls commerçants ; peu à peu, ceux-ci ont commencé à représenter aussi les intérêts des industriels qui ne les ont développés de manière autonome qu'ensuite. Hambourg a toujours été la porte ouverte sur la Chine et l'Asie ; et la ville-Etat est restée le pôle où se concentrent les compétences allemandes sur le commerce extérieur en général et le monde asiatique en particulier. Aujourd'hui, les pouvoirs publics du Land fondent leur politique de compétitivité du site sur cette tradition, n'hésitant pas à mener une stratégie de marketing offensive sur le thème de : Hambourg, «la porte qui ouvre sur le monde » (Hamburg, Das Tor zur Welt). En effet, le port de Hambourg doit en grande partie sa dynamique de croissance aux échanges avec l'Asie. Celle-ci a été littéralement dopée par la chute du Mur et du Rideau de fer qui a conféré à Hambourg une position centrale en Europe et en fait non seulement la plateforme des exportations européennes vers l'Asie, mais également la tête de pont des importations en provenance d'Asie et à destination des marchés est-européens.

Certes, le Land de Hambourg s'est spécialisé dans le commerce maritime avec l'Asie. II n'en est pas moins soumis à une forte concurrence des sites industriels intérieurs, les Länder rivalisant d'ardeur pour développer leurs relations avec la Chine en mettant en avant leurs atouts : la Bavière mise sur Siemens (Munich), le Rhénanie-Palatinat sur BASF (Ludwigshafen), la Rhénanie du Nord-Westphalie sur ThyssenKrupp (Düsseldorf)... Les gouvernements de ces Länder se livrent-ils à une course d'influence en Chine?

Bien sûr, la concurrence entre ces sites et leurs gouvernements est féroce, chacun cherchant à se positionner comme le centre par excellence des relations germano-chinoises et, depuis peu également, comme le pôle des échanges avec I'Inde. Dans ce contexte, l'Ostasiatischer Verein défend sa neutralité. Certes, nos origines nous lient à Hambourg, mais notre mission est d'apporter notre soutien à toutes les entreprises, d'où qu'elles viennent. Et puis, il ne faut pas oublier que si
L'OAV est un réseau d'expertise sur l'Asie, indépendant du politique, et qui joue toujours un rôle de pionnier en Asie

Le port de Hambourg est depuis toujours la 'porte vers l'Asie'

La concurrence entre les Länder se prolonge en Asie 
L'entrée de la Chine dans l'OMC a dopé son économie...

... et fait de la Chine un partenaire à part entière dans le partage mondial du travail les noms de ces quelques grands groupes émergent, la majeure partie des entreprises que nous conseillons est issue du Mittelstand industriel. Nous veillons toujours à défendre les intérêts de l'ensemble des entreprises, sans distinction, car ce n'est qu'ainsi qu'il est possible de présenter efficacement la compétitivité des entreprises allemandes, une compétitivité par nature collective. Mais cette approche se heurte parfois à la compétition interne entre les Länder qui cherchent à développer leur profil à l'international. Assurément, les échanges avec la Chine y gagneraient s'il y avait une meilleure coordination entre les Länder, surtout en ce qui concerne les voyages officiels.

\section{La présence allemande en Chine est géographiquement très concentrée...}

La concentration dans les régions dynamiques de l'est de la Chine n'est pas particulière aux entreprises allemandes. Elle est directement liée au développement industriel de ces régions et à leur réseau d'infrastructures. Cela dit, les entreprises ont commencé à diversifier leurs choix d'implantation, suivant la progression du développement des activités vers l'ouest et le nord-est. Cette extension est aussi une réaction aux difficultés croissantes de recrutement qu'elles rencontrent dans les pôles d'activité de l'est, comme à l'inflation des coûts des facteurs de production. Par contre, l'économie allemande est peu représentée dans le delta de la Rivière des Perles (Zhu Jiang), mais cela s'explique par la structure des activités dans ce bassin spécialisé dans la production de produits bruns et d'électroménager.

\section{L'entrée de la Chine dans l'OMC a dynamisé les économies allemande, européenne et mondiale}

L'histoire des relations germano-chinoises est plutôt mouvementée. Au boom des échanges à la fin du XIX siècle a succédé une période difficile durant les deuX guerres mondiales, l'interruption des relations pendant la Révolution culturelle, renouées à partir de 1979, puis une dynamisation des échanges et l'entrée de la Chine dans l'OMC en 2001. Quel est l'impact de ces phases de tension puis d'apaisement politique sur les échanges commerciaux?

Ces tensions historiques ne jouent plus aucun rôle dans l'état actuel des relations commerciales. Ce qui ne fut pas le cas après la Deuxième Guerre mondiale et durant l'ère de Mao Zedong, où elles étaient entièrement rompues. Or l'Ostasiatischer Verein s'est toujours employé durant ces phases à briser la glace pour renouer les liens. C'est ainsi par exemple que, grâce aussi aux efforts de l'OAV, avait pu être conclu en 1957 le premier accord commercial d'après-guerre entre la République fédérale et la République populaire. Mais notre ère d'échanges ne commence réellement qu'avec la politique d'ouverture engagée sous Deng Xiaoping en 1979. Quant à l'entrée de la Chine à l'OMC, elle marque une étape décisive ; depuis, nous pouvons observer une croissance continue des activités et des échanges, une évolution dont le potentiel semble loin d'être épuisé.

"Guerre, commerce et piraterie - consubstantielle trinité ». C'est sur cette citation extraite du Faust II de Goethe que s'ouvrait le discours tenu lors de l'Agape de l'OAV voici deux ans par le président Horst Köhler. Ce vers prononcé par Mephisto (présenté par H. Köhler comme «le cynique des cyniques ") servait d'argument a contrario à un plaidoyer pour une discussion avec la Chine sur l'adoption de règles visant à organiser le libre-échange. Quels sont les termes en débat?

Les points litigieux: les barrières non douanières, une approche pas toujours transparente ni fair play de la propriété intellectuelle et, plus généralement, une politique protectionniste ne sont nullement une invention chinoise. Bien d'autres nations - dont l'Allemagne - ont fait preuve, au début de l'ère industrielle, d'une semblable créativité pour s'approprier les technologies développées par autrui... Plus récemment, dans les années 1970/80, le Japon et la Corée se sont eux aussi illustrés en la matière. La seule différence avec la Chine d'aujourd'hui est que l'immensité du marché donne une autre ampleur à la problématique dans les 
débats. Le même effet de taille joue dans l'appréciation de la créativité déployée par la Chine dans la mise en œuvre des règles de l'OMC. II suffit de se souvenir de la problématique soulevée voici quelques années, dans le cadre des discussions sur les règles anti-dumping, par les aides publiques à la construction navale coréenne. Rien ne permet donc de désigner la Chine comme le seul mauvais élève. Encore une fois, seul un accord partenarial conclu en toute sérénité, y compris sur des dossiers comme l'accès aux matières premières, est de nature à jeter les bases d'une économie mondiale prospère. Une chose est certaine : dans toutes les discussions menées sur les règles à définir au sein de l'OMC, nous, les Occidentaux, sommes contraints de nous plier à l'évidence, c'est-à-dire de respecter les intérêts comme les besoins de la Chine et de la considérer avec le respect dû à un partenaire à part entière.

Il existe désormais une Chambre de commerce de l'UE en Chine : l'EUCCC, présidée par Jœrg Wuttke, plénipotentiaire de BASF en Chine. Comment s'articulent les stratégies asiatiques de l'Allemagne et de l'UE ? Et la présidence allemande de l'UE a-t-elle permis des avancées?

Une politique asiatique commune à l'Europe peine à s'esquisser. C'est d'autant plus regrettable que si l'UE parlait d'une seule voix et défendait ses intérêts collectifs, elle s'imposerait vis-à-vis de la Chine comme un partenaire d'une autre envergure. Mais l'absence d'une politique commune à l'UE est compréhensible étant donné que les Etats membres continuent de cultiver leurs particularismes dans les domaines politique et économique. Les controverses de ces dernières années sur la prorogation des quotas d'importations de textile/habillement chinois - en violation des accords existants - en sont la parfaite illustration. Quant au processus de la coordination, au sein de l'UE, des accords bilatéraux de libreéchange avec les pays membres de l'Association des Nations d'Asie du Sud-Est (ASEAN), ainsi que la Corée et l'Inde, il se révèle d'une haute complexité. A notre sens, la présidence allemande de l'UE n'a pas apporté d'avancée majeure sur ces dossiers. Quoi qu'il en soit, l'adoption d'une stratégie communautaire unie vis-à-vis de l'Asie sera un processus long et difficile, et dans lequel l'EUCCC joue un rôle clef en matière de lobbying politique.

Quelles sont les barrières non douanières que les entreprises allemandes ressentent comme les plus importantes?

Ce sont les points classiques : transfert de technologies, non respect de la propriété intellectuelle, cahiers des charges locaux imposés en violation des règles de l'OMC dans quelques secteurs considérés comme stratégiques par les autorités chinoises, manque de transparence dans les procédures de certification ou les appels d'offre publics. Les entreprises allemandes évoquent régulièrement ces problèmes dans les instances où elles sont représentées, de même que lors des nombreux débats sur la politique économique, et leur insistance commence à être couronnée de succès. Mais dans nos dialogues avec nos partenaires chinois, nous ne nous contentons pas d'évoquer ces points, nous apportons aussi toujours des arguments permettant d'exposer les avantages que tirent les deux parties d'une coopération fructueuse. Car ce n'est que si nous prenons en considération aussi les intérêts légitimes de la Chine que nous pourrons asseoir durablement un partenariat digne de ce nom. En dehors de ces dialogues où sont abordés les problèmes, chaque entreprise développe bien évidemment sa propre stratégie pour les gérer au quotidien, et d'autant plus aisément que les mêmes problèmes se posent dans d'autres pays.

\section{L'un des problèmes bien connus est le 'transfert' de technologies...}

... il se manifeste sous deux formes : le transfert imposé par les autorités lors de la procédure d'autorisation des sites de production, et le transfert volontaire effectué par les sociétés dans le cadre de leurs obligations contractuelles. Le problème concerne surtout les branches intensives en technologies, où il entraîne des dommages bien plus importants que l'autre aspect de la violation de la propriété intellectuelle : la contrefaçon. La problématique des joint-ventures est plus
L'UE peine à parler d'une seule voix en Chine

La Chine ne respecte guère encore les règles de l'OMC

Fragmenter les savoir-faire pour réduire les risques de piratage des technologies 
Innovation : le seul risque est que l'Allemagne s'endorme sur ses lauriers

Les investisseurs chinois sont les bienvenus en Allemagne

L'Allemagne a su préserver ses parts de marché mondiales créent des têtes de réseau en Asie complexe, car chaque entreprise doit décider au cas par cas si elle accepte ce risque et développer sa propre stratégie pour le limiter. II existe néanmoins un certain nombre de conseils qu'il est vivement recommandé de suivre : veiller à fragmenter les savoir-faire dans l'organisation et les process, y compris dans la gestion de l'outil informatique ; le partenaire allemand doit toujours conserver la maîtrise des compétences clefs. Mais le facteur décisif reste la conscience du danger et l'identification des sources de risque.

Le plan quinquennal chinois 2006-2010 pour les sciences et technologies est-il perçu comme un risque pour une compétitivité allemande qui repose en large partie sur l'innovation?

Le seul risque que puissent courir la compétitivité et l'innovation de l'économie allemande réside dans un éventuel déficit des politiques domestiques d'innovation et de recherche. Le fait que la Chine a lancé un ensemble de programmes destinés à la hisser au rang de nation technologique est désormais largement connu. En revanche, les appréciations sur leur état d'avancement réel divergent. Pour l'Allemagne, cela ne peut être qu'une incitation à préserver notre avantage compétitif et notre capacité d'innovation - et ce, à deux niveaux : grâce à une attitude proactive des entreprises et grâce à la création d'un environnement politique porteur par l'Etat. Mais en aucun cas, nous ne pouvons nous reposer sur nos lauriers - la concurrence ne dort pas.

Un des axes de la politique chinoise est le «spread your wings". Dans quelle mesure les investisseurs chinois sont-ils les bienvenus en Allemagne?

II faut distinguer ici entre d'une part les débats sur les fonds souverains qui ont préoccupé l'opinion ces derniers mois et, de l'autre, la réalité du monde de l'entreprise. Le débat politique autour d'une réforme de la Loi sur le commerce extérieur se nourrissait pour l'essentiel des rivalités politiciennes allemandes et de visées électoralistes. Les milieux économiques traitant avec l'Asie se sont fermement opposés à une limitation des IDE entrants plus stricte que celle qui prévaut dans les secteurs sensibles. Car l'intérêt que portent les investisseurs étrangers, et notamment chinois, au site Allemagne atteste de sa forte attractivité. En ce qui concerne la pratique des entreprises, il existe de nombreux exemples où le rachat d'une société allemande par un investisseur chinois a été un franc succès, puisqu'il a permis de pérenniser ces sociétés et de préserver l'emploi.

La Chine mène une politique renforcée d'échanges avec les pays tiers, s'y érigeant en concurrente des Etats de l'UE comme de l'Allemagne. Cette évolution ne met-elle pas en danger les parts de marché mondiales de l'Allemagne?

L'Allemagne est toujours championne du monde à l'export. Mais il y a plus important que ces palmarès: l'Allemagne est l'une des rares grandes nations industrielles à ne pas avoir perdu de parts de marché sur l'échiquier mondial. II est cependant indéniable que, dans certaines régions du globe, et surtout en Asie, les exportateurs allemands sont exposés à la concurrence impitoyable de leurs homologues chinois, y compris dans des secteurs comme le développement des infrastructures. Or malgré une forte pression au niveau des prix, les produits allemands, qui se caractérisent par leur haute valeur technologique, ont réussi jusqu'ici à s'imposer sur le marché mondial face à la concurrence internationale.

\section{Les principaux défis sont d'ordre culturel}

\section{Quel est le secret des entreprises allemandes pour s'implanter en Chine?}

Les stratégies d'entrée sur le marché chinois différant selon les particularités des entreprises, dont leur taille ou leur appartenance de branche, il n'est pas possible de généraliser leurs approches. On voit s'esquisser néanmoins deux tendances récentes : de plus en plus de sociétés tendent à y créer une filiale indépendante (wholly foreign-owned enterprise), de même qu'un nombre croissant d'entre elles développe une stratégie prenant en considération l'ensemble de l'Asie. Ainsi, au 
lieu de viser isolément chacun des marchés de la région, les entreprises implantent leur production dans un seul pays, ce qui leur permet de s'approvisionner dans les autres pays de la région ou d'y vendre leurs produits. La multiplication des accords bilatéraux ou intra-régionaux conclus au sein de la région Asie a considérablement fluidifié les échanges, ce qui incite les entreprises à y constituer des réseaux globaux d'activités - des achats à la distribution en passant par la production. Cette nouvelle approche globale du marché asiatique élargit leur latitude d'action tout en réduisant les risques, bien qu'elle s'accompagne de contraintes accrues en matière de veille et de planification stratégique. Quoi qu'il en soit, les entreprises allemandes ont définitivement perdu cette naïveté avec laquelle elles s'engageaient en Chine voici seulement dix ans. Et elles ont compris qu'il n'y a pas de recette miracle. Elles sont désormais conscientes qu'un tel engagement demande à être soigneusement planifié et qu'il requiert une solide analyse des risques en termes de coûts/avantages. Et que c'est sur cette base qu'elles doivent développer une stratégie originale pour s'installer en Chine.

Une des difficultés classiques que rencontrent les entreprises allemandes, mais aussi françaises, se situe au niveau des ressources humaines : manque de maind'œuvre qualifiée, forte fluctuation, problèmes de motivation...

... il n'y a là rien de spécifiquement chinois, ce sont les rudes lois du marché : la pénurie de main-d'œuvre qualifiée renchérit les coûts à l'embauche... Une solution consiste bien sûr à former les salariés en interne, mais elle se heurte généralement à la forte mobilité de la main-d'œuvre. Nous conseillons de ce fait aux entreprises allemandes d'offrir une politique intégrée de fidélisation du personnel qui mette l'accent sur les aspects non salariaux comme les perspectives de qualification ou de progression des carrières. Ce qui compte, c'est la perception qu'a chaque salarié de son rôle comme de sa responsabilité sociale dans le collectif qu'est l'entreprise. Les managers allemands doivent tenir compte des différences culturelles avec le pays d'accueil. Or comme l'apprentissage de ces différences et l'adaptation du comportement ne peuvent naître que de l'expérience vécue, il n'y a pas, là non plus, de recette. L'approche est forcément individuelle. II semblerait que la prise de conscience des implications de la globalisation se généralise chez les jeunes Allemands, puisqu'un nombre croissant d'étudiants, toutes filières confondues, s'ouvre aux affaires internationales et particulièrement à l'Asie ; et ils s'expatrient de plus en plus volontiers. Or cette ouverture les sensibilise à la diversité. On commence ainsi à observer que, dans les entreprises allemandes (quel que soit leur statut), le nombre de dirigeants expatriés tend à diminuer. Autrement dit: les sociétés font de plus en plus confiance aux compétences managériales locales.

\section{II fait partie de la culture entrepreneuriale allemande d'offrir une formation initiale et continue. Comment font les sociétés allemandes en Chine?}

Leurs approches sont diverses. Les qualifications qu'elles proposent varient selon les besoins des branches ou ceux des entreprises sur leur marché local. Certaines mènent également des initiatives communes, dont ce Centre de formation professionnelle de Taicang, animé en partenariat par la Chambre de commerce allemande de Shanghai et l'Institut Chien Shiung de technologies. II dispense une formation initiale duale de technicien conforme aux standards allemands et donc sanctionnée par un diplôme reconnu en Allemagne; l'apport de la Chambre de commerce allemande consiste à homologuer les lieux d'apprentissage dans les entreprises d'accueil et à garantir la qualification des formateurs. Le système allemand de formation duale sert là de modèle et jouit d'une haute réputation.

L'approche allemande de la gestion des ressources humaines repose sur le dialogue et le respect mutuel (voir l'entretien avec $U$. Reisach dans REA 84/07). Le mode de gouvernance allemand avec ses hiérarchies plates et le principe de coresponsabilité est-il compatible avec la culture chinoise?

On considère partout, y compris en Chine et dans les autres pays asiatiques, le modèle allemand de GRH avec sa forte implication des salariés et l'exercice des
Fidéliser les salariés en les intégrant dans la communauté qu'est l'entreprise

Les entreprises allemandes exportent la formation duale

Le modèle de gouvernance est difficile à transposer... 
... dans une culture qui ne connaît pas la critique constructive

La représentation des intérêts suit une logique de réseaux

linstitutionnalisée, elle diffère de la culture informelle du guanxi responsabilités quel que soit le niveau hiérarchique comme particulièrement attractif et adapté à la fidélisation des salariés. L'exercice de sa transposition n'en demeure pas moins un défi lorsque les salariés sont habitués, du fait de leur socialisation, à des structures hiérarchiques prononcées impliquant leur soumission à l'autorité du supérieur. Dans une telle culture, il est certes motivant de se voir confier une part d'initiative et de responsabilités, mais cela s'accompagne aussi d'une exigence inaccoutumée et donc déstabilisante. Cette autonomie de chacun des collaborateurs n'est pas tant l'expression de cette co-responsabilité institutionnelle (Mitbestimmung) exercée via le Conseil d'entreprise (Betriebsrat) et régie par le droit du travail qu'elle ne reflète l'impératif de relations de confiance dans les process exigeant un travail en équipe. Cela dit, le modèle allemand de la Mitbestimmung contribue assurément à fonder une approche de l'entreprise comme un collectif où patron et salariés engagent leur responsabilité pour œuvrer à sa prospérité. II entretient de la sorte l'identification du salarié avec son entreprise.

Il est vrai que, par contraste, le principal défi pour un manager allemand qui découvre la Chine consiste à gérer des équipes de salariés qui se contentent d'exécuter les consignes sans s'interroger sur leur bien-fondé et sans jamais chercher à adapter leur tâche à des situations variables. La culture d'entreprise chinoise accorde moins d'importance à la réactivité de chacun et fait moins appel à la créativité pour résoudre un problème. Elle ne connaît pas, en effet, ce trait foncier de la culture d'entreprise allemande qu'est la pratique de la contradiction, de la critique constructive au sein d'une équipe dans un processus collectif de prise de décision, que ce processus soit vertical, traversant tous les échelons hiérarchiques, ou qu'il soit horizontal, c'est-à-dire qu'il associe en réseau les diverses directions ou divisions concernées, et tout particulièrement la R\&D. Pour le dire autrement, dans une entreprise allemande, du fait de son implication, chaque salarié doit respecter dans l'approche de son travail non seulement les intérêts de ses homologues dans les autres directions, mais aussi les impératifs de l'entreprise considérée comme un tout. Transmettre cette vision et chercher à l'impulser au quotidien relève du défi...

\section{Une des caractéristiques de l'économie allemande est son fonctionnement en réseau de partenariats. Ce modèle est-il adapté à la société chinoise ?}

D'abord un mot sur le dispositif de la représentation allemande. Les chambres de commerce allemandes en Chine (les $\mathrm{AHK}$ ) constituent le réseau institutionnel de la représentation des entreprises allemandes dans la région. La Fédération allemande de l'industrie (BDI) et l'Ostasiatischer Verein n'y ont pas de bureau propre, puisqu'elles sont représentées par les AHK. Quant aux German Centres de Shanghai et de Pékin, ils assument une fonction de tête de réseau en Chine. Membres d'un réseau mondial, ils sont le fruit d'une coopération entre un fonds d'investissement immobilier privé, diverses banques publiques régionales (BadeWurtemberg en tête), des fédérations comme la DIHK, le BDI ou la VDMA (construction mécanique), ainsi que divers ministères du Bund ou des Länder. Ces German Centres sont nés à l'initiative de Berthold Leibinger, qui dirigea le groupe Trumpf (construction mécanique) pendant près de trente ans et qui souhaitait permettre aux PME allemandes, grâce à ces réseaux, de se développer à l'international à risques et coûts raisonnés. II était parvenu à convaincre du projet les banques publiques de son Land (le Bade-Wurtemberg), sa fédération professionnelle et les pouvoirs publics. Ces centres offrent aux entreprises pour l'essentiel des prestations immobilières, les conseillant et prenant à leur charge le risque de la location de bureaux. Cette activité génère par nature une logique de réseau qui fait des German Centres des lieux de contact et de rencontre.

Or si ces structures répondent au besoin d'échange des sociétés allemandes dans la région, elles sont totalement étrangères à la culture chinoise des réseaux (guanxi). Mais elles permettent le partage d'expériences sur les meilleures manières d'aborder ce guanxi qui se traduit par la culture de réseaux informels de 
contacts au sein des institutions, des entreprises, de la société, etc. Cette approche est par définition étrangère à une culture allemande plus formaliste et où les réseaux de partenariat impliquent une claire répartition des rôles respectifs.

\footnotetext{
«En Chine aussi, on porte une haute considération à ces vertus considérées comme allemandes : honnêteté, ponctualité, sens de de la justice, de la vérité, et ouverture d'esprit », écrit un observateur de la Chine (Kausch, 2007). Qu'en est-il réellement?
}

Le fait que ces vertus prêtées aux Allemands aient une image positive ne signifie pas que leurs partenaires chinois se les approprient. S'ils les tiennent en haute estime, cela relève d'une certaine idéalisation ou, plutôt, de la généralisation hâtive d'une expérience heureuse, puisque ces 'vertus' se traduisent dans le concret par une grande prévisibilité et une tout aussi grande fiabilité. En ce sens, elles confèrent à l'Allemagne un avantage en termes d'image. Mais le revers de la médaille est que cette bonne réputation ne facilite pas aux managers allemands la compréhension de la culture des affaires foncièrement différente chez leurs partenaires chinois. Que, le lendemain de la conclusion d'un accord, celui-ci puisse être l'objet d'une renégociation leur donne du fil à retordre. De même, ils ont beaucoup de mal à accepter l'idée que, dans l'approche chinoise, un contrat même écrit n'est jamais que la base d'un accord dont les termes sont adaptés en permanence à une donne évolutive. Ici, comme dans toute constellation marquée par des différences culturelles, le secret de la réussite consiste à ne pas déroger à sa manière habituelle de faire, mais tout en cherchant à comprendre les règles qui guident l'action du partenaire. Cela dit, la connotation positive de l'image des entreprises allemandes repose aussi et avant tout sur un présupposé lié à la qualité des produits made in Germany, réputés pour leur haute teneur en technologies et leur durée de vie. Or dans la réalité des affaires, ces critères-là comptent plus, il me semble, que les facteurs culturels ou leur connotation, aussi positive soit-elle.

\section{Aborder la Chine et la mondialisation « avec pragmatisme »}

\section{L'Empire du Milieu fascine les Européens. Qu'est-ce qui incite une entreprise alle- mande à faire comme dans ce conte des Frères Grimm et à « s'en aller apprendre la peur » en Chine?}

Je ne présenterais pas les choses ainsi ; les entreprises allemandes n'ont pas « appris la peur » en Chine. Les peurs qui sous-tendent le discours sur la Chine dans nos sociétés sont nourries par ceux qui ignorent ce pays et qui se sentent de ce fait menacés par sa taille, sa culture très différente et son immense dynamisme économique. Les entreprises allemandes ne se meuvent pas dans un monde imaginaire ; elles ont à affronter des défis très réels dans un environnement hautement concurrentiel où soit elles réussissent soit elles échouent. Leur sort relève de la part de risque inhérent à toute activité, et c'est uniquement sous cet angle qu'il faut considérer la question, surtout dans les cas où la culture des affaires propre aux partenaires chinois crée, parfois, des situations imprévisibles. La culture allemande n'aime pas l'imprévisibilité, mais la réalité est ainsi, et il faut l'aborder avec pragmatisme. "L'Asie change la face du monde ", affirmait Horst Köhler dans son discours de-
vant l'OAV. Qu'est-ce que la Chine apporte à l'Allemagne?

Pour la société allemande et, par-delà, l'ensemble de l'Europe, la Chine est synonyme de la nécessité d'affronter la globalisation en adaptant notre vision du monde et notre modèle de société aux profonds changements qu'elle induit. Le plus grand défi pour nos sociétés comme pour notre modèle politique consiste à faire comprendre aux Allemands et aux Européens que nous vivons une époque où les équilibres politiques et économiques sont en train de se modifier et qu'il nous revient d'adopter une attitude positive et proactive face à ce processus afin de préserver l'avenir de nos intérêts et de notre prospérité.
Image positive des Allemands et, plus bien plus important, du made in Germany
Développer une attitude proactive face aux mutations mondiales 
Un impératif absolu : abolir les frontières mentales
Ce même discours était un plaidoyer pour l'institution d'un ordre mondial dont les règles « reposent sur le respect et la reconnaissance mutuelle, sur la coopération et le développement durable ". Quelles recommandations formule l'OAV en tant que représentation des milieux économiques pour l'action du monde politique?

Abolir les frontières mentales, supprimer les entraves au libre-échange et à l'investissement. L'Allemagne est et doit rester un partenaire économique ouvert au monde et aux échanges. Elle doit s'affirmer encore plus dans son rôle de pionnier dans le développement de technologies durables et se positionner en partenaire de l'Asie dans ces défis que nous ne pouvons affronter qu'ensemble. Voilà le message que nous cherchons à faire passer au sein de l'UE et dans l'espace public allemand.

\section{Si l'économie allemande pouvait faire un vœu, lequel ?}

Notre souhait le plus cher est de voir cesser le discours peu différencié sur la Chine.

Propos recueillis et traduits par Isabelle Bourgeois

\section{Indications bibliographiques}

STÄRK M. (ed), Wirtschaftshandbuch Asien-Pazifik 2007/2008, Hamburg 2007

L'OAV publie notamment la revue mensuelle OAV Report et coédite le mensuel Asia Bridge en coopération avec le groupe Dow Jones.

Voir aussi :

BOURGEOIS I., "La place de l'Allemagne dans l'économie mondiale », et BouRGEOIS I., LASSERRE R., "Les PME allemandes : acteurs de la mondialisation ", Regards sur l'Economie Allemande, $n^{\circ} 83 / 2007$

"Culture d'entreprise: 'le piège de l'américanisation'. Un entretien avec Ulrike Reisach », Regards sur l'Economie Allemande, n`84/2007

Eberstein B., Der Ostasiatische Verein 1900-2000, Hambourg, 2000

FARGEL Y., MüLleR-Seitz G., Lu L., “ Netzwerke in China », in Dossier : « Interkulturelles Management ", Personal, mai 2005

GABEL M., "Chine, Inde, Brésil: les choix des enterprises allemandes », Regards sur l'Economie Allemande, $\mathrm{n}^{\circ} 70 / 2005$

KaUSCH U., China-Pioniere. Unternehmen berichten von ihren Erfolgen im Reich der Mitte, Francfort, 2007

MEIER R., REISACH U. (eds), Aufbruch im Land des Drachen. Arbeiten und Leben in China zwischen Konfuzianismus, Sozialismus und Globalisierung, Gernsbach, 2008

"'Morale et profit ne sont pas incompatibles'. Un entretien avec Dominik Enste ", Regards sur l'Economie Allemande, $n^{\circ} 84 / 2007$

ReinHARD M., TAUBe M., WASmer C., "Technologiepolitik in China - Gefahr für die Wettbewerbsfähigkeit Deutschlands? ", ifo-Schnelldienst, 23/2007

ReISACH U., TAUber T., YuAN X., China - Wirtschaftspartner zwischen Wunsch und Wirklichkeit. Ein Handbuch für Praktiker, $4^{\mathrm{e}}$ édition revue et augmentée, Heidelberg, 2007

RothACHER A., Mythos Asien? Licht und Schatten einer Region im Aufbruch, Munich, 2007

WEINACHTER M., «Hambourg : région métropole européenne et site portuaire international », in BouRGEoIs I. (dir.), Allemagne : compétitivité et dynamiques territoriales, Coll. Travaux et Documents du CIRAC, Cergy-Pontoise, juillet 2007

www.oav.de et www.asien-pazifik-ausschuss.de. 